\title{
Zum 80. Geburtstag von Prof. Dr. Georg Mattheß - Sonderbeiträge
}

\author{
Margot Isenbeck-Schröter
}

Eingang des Beitrages: 11.9.2012 / Online veröffentlicht: 1.11.2012

(C) Springer-Verlag Berlin Heidelberg 2012

Liebe Leserinnen und Leser,

nachdem ich um die Jahrtausendwende herum einige Jahre Editorin der Zeitschrift Grundwasser gewesen war, durfte ich nun als Gasteditorin die vorliegende Dezemberausgabe 2012 editieren. Sie ist anlässlich seines 80 . Geburtstages im Februar 2012 Herrn Prof. Dr. Georg Mattheß gewidmet.

\section{Lieber Georg,}

mit dieser Ausgabe möchten wir Dir herzlich zu Deinem Geburtstag gratulieren und Dir unsere Aufwartung machen. Wir, das sind einerseits das Team der Zeitschrift Grundwasser - besonders nennen möchte ich hier die ehemalige Kieler Doktorandin Ruth Kaufmann-Knoke - und der Vorstand der FH-DGG, andererseits Deine wissenschaftlichen Nachfahren, die jetzt noch aktiv an Universitäten oder in Verwaltungen tätig sind und zu denen ich mich auch zählen darf. Leider fehlen inzwischen einige geschätzte Kollegen, da sie viel zu früh verstorben sind. Ich denke da vor allem an Henning Holthusen, Dietmar Schenk und Asaf Pekdeger. Mit meiner Zeit in Deiner Arbeitsgruppe von 1982 bis 1990 ist die Erinnerung an Dietmar und Asaf eng verbunden. Es war eine sehr ereignisreiche und kreative Zeit, in der Du mit Deiner Arbeitsgruppe die Hydrogeologie in Deutschland weitreichend und nachhaltig mit gestaltet hast. Thematisch lag Dir vor allem der Bereich der Hydrogeochemie am Herzen. Durch die vielschichtigen Projekte zur hydrogeochemischen Grundwasserentwicklung, zum Transport von Bakterien und Viren sowie Schadstoffen oder später dann durch das von uns beiden gemeinsam konzipierte Pes-

M. Isenbeck-Schröter $(\varangle)$

Universität Heidelberg, Heidelberg, Deutschland

E-Mail: Margot.Isenbeck@geow.uni-heidelberg.de

tizidprojekt hast Du die Grundwasserchemie und Modellierung in den Mittelpunkt des Interesses gesetzt.

Anekdoten aus der Kieler Zeit kenne ich viele. Besonders gern erinnere ich mich an Deine Rückkehr aus Waterloo nach Deinem Sabbatical dort. Deine engen Mitarbeiter bekamen IBM-Computer auf den Schreibtisch - damals eine Innovation aber auch eine echte Investition. So begannen wir zu "modellieren", sei es geochemisch unter der Anleitung von Horst Schulz und Asaf Pekdeger, die schon mit Commodore vorgearbeitet hatten, oder auch hydraulisch. Hier wurde der noch junge Wolfgang Kinzelbach für eine Unterrichtswoche nach Kiel geholt, um die Arbeitsgruppe mit den Prinzipien der hydraulischen Modellierung und der Transportmodellierung vertraut zu machen. Wir beide teilten uns damals einen Rechner und versuchten uns gemeinsam an den Kinzelbach-Übungen.

Noch heute bin ich ein großer Fan geochemischer Modellierungen, wie unser Heidelberger Beitrag zu einem Fallbeispiel aus Nordwestdeutschland zeigt. Sven Rumohrs Artikel stellt die Brücke zwischen Deiner Zeit am Wiesbadener Landesamt und der Kieler Zeit dar. Die Auseinandersetzung mit der „Wünschelrute“ hat Dich immer wieder beschäftigt. Maria Schafmeister beschreibt Deinen Werdegang aufbauend auf Deinen Aufzeichnungen. Traugott Scheytt und Coautoren erinnern indirekt an Deine Arbeiten gemeinsam mit Herrn Golwer am Monte Scherbelino. Jürgen Schröter greift als Coautor die Thematik der Fuzzy-Logik auf, die Jan Piotrowski in Deiner Arbeitsgruppe in Kiel etabliert hat.

Lieber Georg, ich hoffe Du bist auch stolz auf Dein Lebenswerk und auf die Spuren, die geblieben sind. In diesem Sinne wünsche ich Dir viel Vergnügen beim Lesen Deines Grundwasser-Heftes!

Deine Margot Isenbeck-Schröter, Universität Heidelberg 